\section{Implicit Control}

\author{
Paolo Rocco \\ Politecnico di Milano, Dipartimento di \\ Elettronica, Informazione e Bioingegneria, \\ Milano, Italy
}

\section{Definitions}

This chapter discusses implicit control for robot manipulators. Although the terminology is not homogeneous in the literature, here we assume that an implicit control is a way to control the force arising when the end effector makes contact with the environment, where the force control loop is closed around inner position/velocity loops. As opposed, an explicit control scheme assumes that the output of the force controller is directly in terms of the motion control actuation variables (torques or forces, depending on the nature of the joints).

\section{Overview}

Robots make contact with the environments for a number of reasons, the most common being the accomplishment of some tasks, like assembly or machining a certain part. In such contact situations, it is desirable to control the interaction, either by directly controlling the contact force to certain values or by imposing appropriate dynamic behaviors to the relation between the contact force and the resulting motion of the robot (impedance control). In both cases the output of the force (or impedance) controller can be expressed in terms of joint torques. Unfortunately transmission mechanisms used in robotics, and in particular in industrial robotics, are affected by several harmful dynamic effects (like friction, backlash, and compliance), which make an accurate generation of the joint torque problematic.

A more common implementation of force control scheme thus relies in inner position/velocity loops, whereby the output of the force controller is the target position/velocity for the inner loops. This arrangement (called implicit force control) simplifies the implementation of the force control for industrial robots, since the industrial architecture of the motion control system is in any case built around high bandwidth joint position controllers. The only openness left for other control loops is thus in terms of modification in real time of the setpoints of such position/velocity loops, the modification of the joint torques being not allowed.

Implicit force control has been proposed first in De Schutter and Van Brussel (1988). Rocco et al. (1997) discussed the stability issues of implicit force control for robots in contact with stiff surfaces. Applications of implicit force control have been presented in Kröger et al. (2004), Osypiuk et al. (2006), and Winkler and Suchy (2016). Rossi et al. $(2014,2016)$ discussed the effects of joint and link elasticities in the performance of implicit force control, while Parigi 
Polverini et al. (2017) applied advanced methods based on set invariance to design an implicit force controller.

\section{Key Research Findings}

\section{A One Degree of Freedom System}

The concept of implicit force control is best understood making reference to a one degree of freedom system. Consider then a single mass in contact with the environment (Fig. 1), with a position control system closed around. Assume an elastic behavior of the environment, and let $x$ be the position of the mass, $x_{d}$ the desired value of such position, $u$ the actuation force, and $f_{e}$ the reaction force at the contact.

The idea of the implicit force control is to measure the force $f_{e}$, compare it to a setpoint value $f_{d}$, and elaborate a control signal which is applied through the position setpoint $x_{d}$ (Fig. 2). In this arrangement, the position control is purely instrumental to the force control outer loop, while in a more general scenario of a multi-degree of freedom robot, it is in charge of the control of the motion of the manipulator in the directions unconstrained by the environment.

For the design of the force controller, the classical approach is to consider the same closedloop transfer function of the position control as in free motion, thus assuming that the effect of the reaction force is well rejected by the (high bandwidth) position control system (Fig. 3). In a first approximation, such closed-loop transfer function can be expressed through a second- order filter with unitary gain, bandwidth $\omega_{p}$, and damping ratio $\zeta_{p}$ (typically $0.7<\zeta_{p}<1$ ):

$$
h(s)=\frac{\omega_{p}^{2}}{s^{2}+2 \zeta_{p} \omega_{p} s+\omega_{p}^{2}}
$$

The force controller can then be closed compensating the stiffness constant $k_{e}$ of the environment and adopting a purely integral controller, tuned to achieve a desired bandwidth (necessarily smaller than the bandwidth $\omega_{p}$ of the position control system). The bandwidth of the force control system can be increased adding a proportional action in the controller (PI controller).

On the other hand, solutions where the force controller does not exhibit a low-pass filtering action, as well as solutions where a derivative of the force measurement is required, are not advisable (due to uncertainties in the resulting bandwidth and to noise amplification, respectively).

\section{Implicit Control in Case of Stiff Environment}

The design of the force controller as described above is valid for a rigid mechanism and an elastic environment, meaning that the stiffness of the mechanism has to be significantly larger than the stiffness of the environment. However, robots are usually in contact with stiff environments (refer to technological operations like deburring or grinding of metal surfaces). In the limit as the environment becomes infinitely stiff, the scenario changes significantly: since the position is constrained by the environment, the position loop gets open, while the relation between the
Implicit Control, Fig. 1

Position-controlled one degree of freedom system in contact with the environment

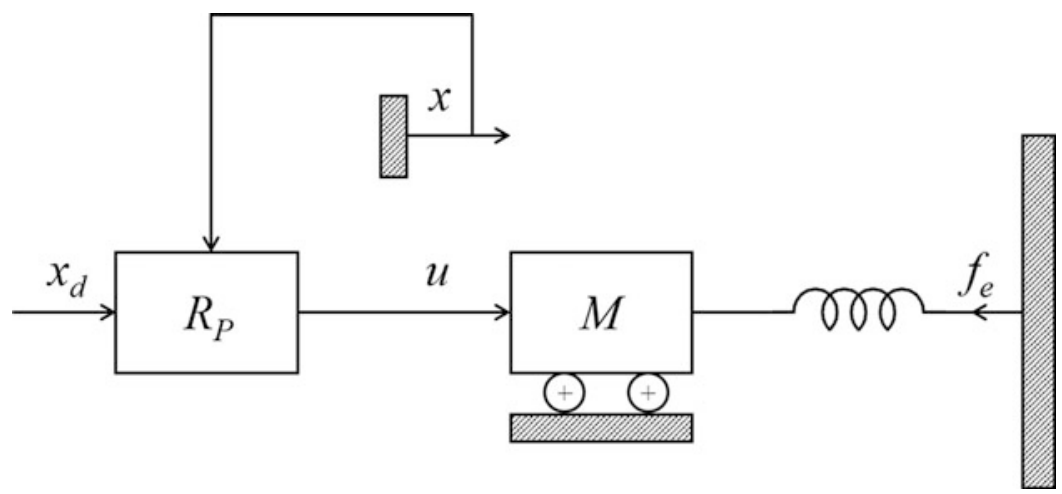


Implicit Control, Fig. 2

Implicit force controller

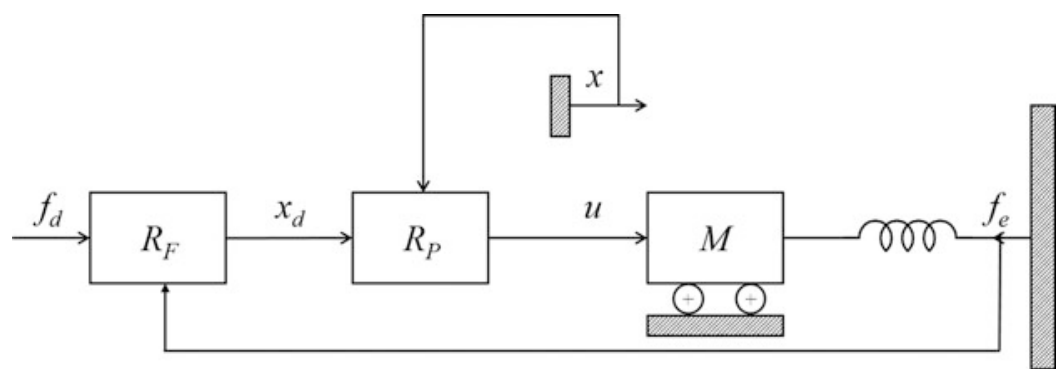

Implicit Control, Fig. 3

Block diagram of the implicit force controller


Implicit Control, Fig. 4 Implicit force control scheme in case of stiff environment

actuation force $u$ and the reaction force $f_{e}$ of the environment is algebraic (infinitely fast) with unitary gain. This means that the force controller has to be tuned making reference to a transfer function which corresponds to the open-loop transfer function $R_{P}(s)$ of the position controller.

Assuming that the position controller is implemented through a standard PID,

$$
R_{P}(s)=\frac{K_{D} s^{2}+K_{P} s+K_{I}}{s}
$$

the force controller can compensate for the zeros of such controller

$$
C(s)=\frac{1}{K_{D} s^{2}+K_{P} s+K_{I}}
$$

and then close the loop with a PI regulator; see Fig. 4. Since the loop transfer function in this case amounts just to

$$
L(s)=\frac{K_{P F} s+K_{I F}}{s^{2}},
$$

where $K_{P F}$ and $K_{I F}$ are the proportional and integral gains of the controller, respectively, tuning of the two controller gains is trivial.

The situation is more involved when the mechanism under control presents significant compliance at the joint between the motor and the link. In Rocco et al. (1997), it is shown that, in this case, when the environment is infinitely stiff, the integral action combines with the compliance of the joint, giving rise to a low frequency mode that needs to be compensated by the force controller.

The control scheme is sketched in Fig. 5, where $K$ is the compliance at the joint. After compensation of the low frequency mode, through the transfer function

$$
C(s)=\frac{\left(K+K_{P}\right) s+K_{I}}{K\left(K_{D} s^{2}+K_{P} s+K_{I}\right)}
$$

the force controller can be closed with a simple integral controller. In Rocco et al. (1997), it is also shown that the stiffer the environment is, the more stable the force control system is, when using an integral controller (the opposite is true when using a proportional-derivative controller). 
Implicit Control, Fig. $\mathbf{5}$

Implicit force control scheme in case of stiff environment and compliant joint

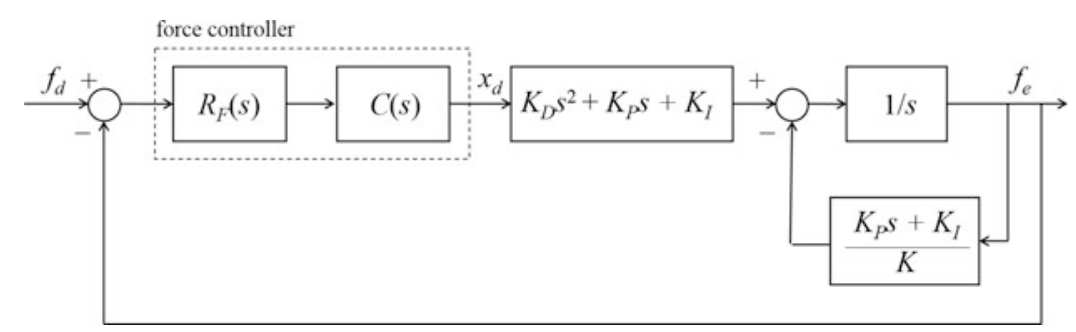

$q$

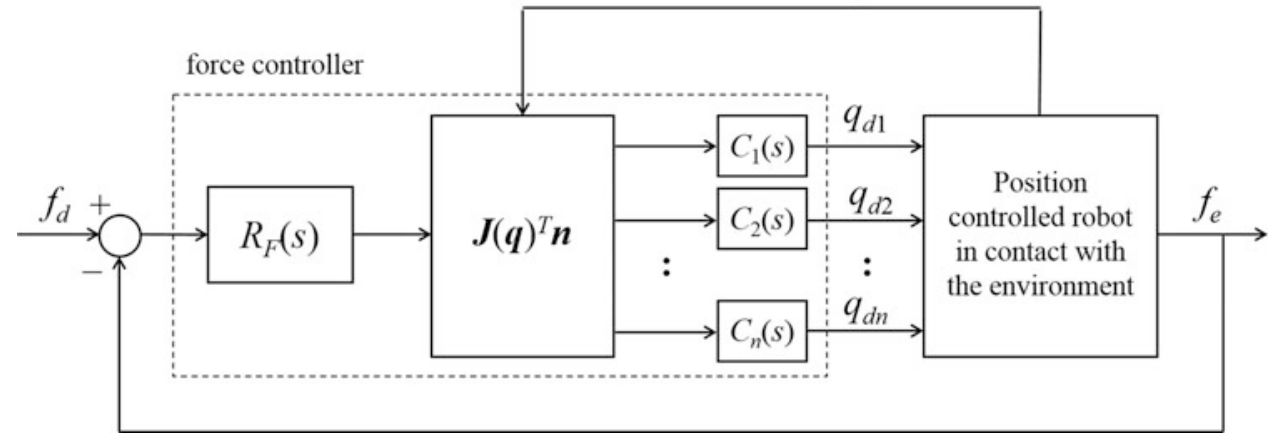

Implicit Control, Fig. 6 Implicit force control scheme for a multi-DOF manipulator, in case of stiff environment and compliant joints

\section{Extension to a Multi-degree of Freedom Robot}

The discussion of the previous sections can be easily extended to a multi-degree of freedom system. We neglect here the dynamic model of the robot, assuming that the high bandwidth inner position loops provide enough decoupling effect. Assuming a point contact with a stiff surface whose normal is identified by the unit vector $\mathbf{n}$, the control scheme in Fig. 5 can be extended as in Fig. 6. The action of the force controller is projected onto the normal to the surface and then converted in joint space through the geometrical Jacobian $\mathbf{J}$ of the manipulator. The compensators $C_{i}(s)$ of the position controller dynamics act on the single joints (see also Ferretti et al. 1997). Notice that with the arrangement sketched in Fig. 6, the force controller acts only in the direction where motion is constrained by the environment, without disturbing the motion control system in the remaining directions.

\section{Examples of Applications and Future Directions of Research}

The use of force control, and specifically of implicit force control, is becoming increasingly interesting for industrial, as well as non-industrial, applications. Whenever a contact with the environment is established, the accurate control of the interaction force can highly improve the performance of the operation. Precise assembly operations and robotized machining of mechanical parts are just examples of industrially relevant situations.

Research can follow up this renewed interest in implicit control along several lines, among which:

- Modern robots (especially collaborative ones) are lightweight, and the paradigm of rigid robotics might prove to be inadequate to represent the complexity of the contact situation. 
Methods that can online estimate the effective stiffness of the robot in the direction of the contact are particularly relevant to allow for better tuning of the implicit force controller. Learning methods are of special interest as well.

- The implicit force controller as described in these notes is a classical linear controller. An active research line is to address the force control problem with modern nonlinear control methods. Force regulation with improved settling performance and absence of force overshoots can in fact be achieved with a constrained control approach exploiting the framework of invariance control (see, e.g., Parigi Polverini et al. 2017).

\section{Cross-References}

\author{
- Force Control \\ - Hybrid Force and Position Control
}

\section{References}

De Schutter J, Van Brussel H (1988) Compliant robot motion II. A control approach based on external control loops. Int J Robot Res 7:18-33
Ferretti G, Magnani G, Rocco P (1997) Towards the implementation of hybrid position/force control in industrial robots. IEEE Trans Robot Autom 13:838-844

Kröger T, Finkemeyer B, Neuck M, Wahl F (2004) Adaptive implicit hybrid force/pose control of industrial manipulators: compliant motion experiments. In: Proceedings of IEEE/RSJ international conference on intelligent robots and systems, pp 816-821

Osypiuk R, Kröger T, Finkemeyer B, Wahl F (2006) A two-loop implicit force/position control structure, based on simple linear model: theory and experiment. In: Proceedings of IEEE international conference on robotics and automation, pp 2232-2237

Parigi Polverini M, Nicolis D, Zanchettin A, Rocco P (2017) Implicit robot force control based on set invariance. IEEE Robot Autom Lett 2:1288-1295

Rocco P, Ferretti G, Magnani G (1997) Implicit force control for industrial robots in contact with stiff surfaces. Automatica 33:2041-2047

Rossi R, Bascetta L, Rocco P (2014) Implicit force control for an industrial robot with flexible joints and flexible links. In: Proceedings of IEEE/RSJ international conference on intelligent robots and systems, pp 47424749

Rossi R, Fossali L, Novazzi A, Bascetta L, Rocco P (2016) Implicit force control for an industrial robot based on stiffness estimation and compensation during motion. In: Proceedings of IEEE international conference on robotics and automation, pp 1138-1145

Winkler A, Suchy J (2016) Explicit and implicit force control of an industrial manipulator - an experimental summary. In: Proceedings of IEEE international confernce on Meth. Mod. in Autom. Rob., pp 19-24 